\title{
A aplicação do DOI para publicações no Portal de Periódicos da UFGD e a busca por novos indexadores
}

\author{
Givaldo Ramos da Silva Filho \\ Universidade Federal da Grande Dourados \\ givaldofilho@ufgd.edu.br \\ Rodrigo Garófallo Garcia \\ Universidade Federal da Grande Dourados \\ Cynara Almeida Amaral \\ Universidade Federal da Grande Dourados \\ Wanessa Gonçalves Silva \\ Universidade Federal da Grande Dourados \\ Raquel Correia de Oliveira \\ Universidade Federal da Grande Dourados \\ Jessica Sotolani Manfré \\ Universidade Federal da Grande Dourados
}

\section{Como citar:}

SILVA FILHO, G. R. el al. A aplicação do DOI para publicações no Portal de Periódicos da UFGD e a busca por novos indexadores. In: ABEC MEETING, 2, 2018, São Paulo. Anais... São Paulo: Associação Brasileira de Editores Científicos, 2018. p. 1-6.

http://dx.doi.org/10.21452/abecmeeting.2018.158

\section{RESUMO}

Este artigo tem o objetivo de apresentar um estudo de caso sobre o trabalho que foi desenvolvido pela Editora da Universidade Federal da Grande Dourados (UFGD), a qual administra o Portal de Periódicos UFGD (PPUFGD), visando melhorar a qualidade e a divulgação das publicações científicas da instituição. Trata-se de uma experiência em que a Editora da UFGD buscou, através de processos administrativos e procedimentos tecnológicos, aumentar a divulgação de seus periódicos com a obtenção do Digital Object Identifier (DOI), via CrossRef/Publisher International Linking Association (PILA) - agência oficial de registro do DOI, para os periódicos da UFGD. Também foi feita a adequação dos periódicos do Portal às exigências de diversas bases de dados e diretórios nacionais e internacionais (indexadores), dentre eles o Directory of Open Access Journals (DOAJ).

Palavras-chave: Portal de Periódicos UFGD. DOI. Indexadores.

\section{ABSTRACT}

This paper aims at presenting a case study about the work developed by the Publisher of Federal University of Grande Dourados (UFGD) regarding the UFGD Portal of Scientific Journals. The UFGD Publisher's 
main goal was to improve the journals' quality and disseminate the university scientific production. It tells the UFGD Publisher experience with Digital Object Identifier (DOI) acquisition through CrossRef/Publisher International Linking Association (PILA) and discusses the administrative and technological procedures involved in this process. This paper also brings information on how the journals available on UFGD Portal were adapted to the requirements of several national and international databases and directories (indexers), such as the Directory of Open Access Journals (DOAJ).

Keywords: UFGD Portal of Scientific Journals. DOI. Indexers.

\section{INTRODUÇÃO}

Criado como um padrão para identificar documentos e objetos na internet, de acordo com Brito et al. (2015, p. 9), o Digital Object Identifier (DOI):

[...] é um sistema que engloba diferentes subsistemas para o depósito de metadados e a resolução dos nomes DOI. Consiste em um par identificador e metadados, em que os metadados podem ser recuperados a partir do identificador. O DOI é um identificador persistente, único e publicado que gestores de conteúdo vinculam a objetos físicos ou digitais, possibilitando ofertar serviços e garantir a propriedade intelectual, principalmente para objetos digitais disponíveis na Internet.

Nesse sentido, com o objetivo de potencializar a divulgação das publicações da Universidade Federal da Grande Dourados (UFGD), a Editora da UFGD (EdUFGD) participou de diversos encontros e treinamentos, como, por exemplo, do XV Encontro de Editores Científicos (ENEC) em 2015, na cidade de Florianópolis-SC, e do IX Workshop de Editoração Científica em 2016, em São Pedro-SP, ambos eventos organizados pela Associação Brasileira de Editores Científicos (ABEC). Nesses encontros, os servidores da EdUFGD receberam treinamentos e informações sobre como solicitar e atribuir o DOI para os periódicos e também como adequar as informações, os metadados e o layout de cada revista aos critérios mínimos exigidos pelos diversos diretórios e bases de dados nacionais e internacionais.

O Portal de Periódicos da UFGD (PPUFGD) adotou o Open Journal Systems (OJS) em 2007 e, atualmente, hospeda 16 periódicos (Quadro 1) de diversas áreas do conhecimento, sendo administrado pela Editora da UFGD, com o apoio da Biblioteca Central (BC) e da Coordenadoria de Informática (COIN). O PPUFGD tem o objetivo de integrar e disponibilizar os periódicos acadêmicos e científicos desenvolvidos pelas unidades acadêmicas e por programas de pós-graduação da instituição. A coordenação geral do Portal é de responsabilidade do coordenador editorial da própria Editora. O suporte aos usuários do portal é feito por dois técnicos-administrativos e uma estagiária, e a tradução para o inglês de alguns elementos e metadados contidos no Portal é feita por uma tradutora e intérprete, todos lotados na Editora. O Portal conta também com o serviço de revisão ortográfica e normalização de duas revisoras exclusivamente para informações do Portal, pois a revisão do conteúdo dos periódicos é de responsabilidade dos editores de cada revista. O suporte em tecnologia da informação é feito por um técnico da Coordenadoria de Informática da própria instituição e os profissionais da Biblioteca Central dão suporte técnico relativo à gestão da informação. 
Quadro 1 - Revistas do Portal de periódicos da UFGD.

\begin{tabular}{c|c}
\hline TÍTULO & ÁREA DO CONHECIMENTO \\
\hline Agrarian & Ciências Agrárias \\
\hline Arredia & Letras \\
\hline Ead e Tecnologias Digitais na Educação & Educação à distância \\
\hline Educação e Fronteiras Online & Educação \\
\hline Entre-Lugar & Geografia \\
\hline Fronteiras & História \\
\hline Horizontes & Educação \\
\hline Monções & Relações Internacionais \\
\hline Movimentação & Antropologia \\
\hline Nanduty & Letras \\
\hline Raído & História \\
\hline Realização & Geografia \\
\hline Revista Eletrônica História em Reflexão & Direito \\
\hline Revista da ANPEGE & Educação Matemática \\
\hline Videre & \\
\hline Tangram & Interdisciplinar (Revista de Extensão) \\
\hline
\end{tabular}

Fonte: Elaborado pelos autores, 2018.

\section{PROCEDIMENTOS PARA A OBTENÇÃO DO DOI}

A aquisição do DOI via Crossref é fundamental para potencializar a visibilidade e o acesso a publicações científicas. Em fevereiro de 2018, a ABEC publicou uma entrevista com os especialistas Suely de Brito Clemente Soares e Gildenir Carolino Santos sobre a importância de se obter o DOI. O professor Gildenir Santos, do Portal de Periódicos da Unicamp, relata que:

Da mesma forma da importância de citar o trabalho para reconhecimento, com a atribuição do DOI o trabalho também ganha visibilidade internacional. Há também a persistência do link de não ser removido de hipótese alguma, mesmo se for removido de determinado servidor. O link mantémse persistente e não há quebra dele na recuperação da publicação na Internet. (ABEC, 2018).

O processo de atribuição de DOIs às publicações científicas do PPUFGD foi iniciado em março de 2018 com o intuito de adequar os periódicos publicados pela UFGD a esse conceito. No mesmo ano, foi firmado um convênio (Represented Member Agreement) entre a CrossRef e a UFGD por intermédio da ABEC, à qual a EdUFGD é associada desde 2009. Desta forma, a EdUFGD pôde receber uma orientação muito precisa por parte dos técnicos dessa associação, e os identificadores digitais começaram a ser aplicados aos periódicos da instituição. Fomos informados pela ABEC sobre o prefixo DOI (10.30612) atribuído aos periódicos do PPUFGD e recebemos um login e uma senha de acesso para que a EdUFGD começasse a depositar os DOIs diretamente no site da CrossRef. Um processo administrativo institucional foi aberto e um empenho estimativo foi gerado para a realização de pagamento do serviço de atribuição e de validação de DOIs a cada trimestre. As vantagens de tal procedimento são o fato de não haver a necessidade de pagamento de anuidade à CrossRef e também a possibilidade de realizar o pagamento 
dos serviços diretamente à ABEC.

Em relação à questão técnica, a princípio, realizamos uma pesquisa para obter informações sobre quais procedimentos deveriam ser adotados para a obtenção dos DOIs. Utilizamos como material de apoio o Manual do usuário do Digital Object Identifier (DOI $尺)$, que é resultado do trabalho conjunto entre a $A B E C$ e o Instituto Brasileiro de Informação em Ciência e Tecnologia (IBICT). Esse manual foi muito útil para sabermos qual era a melhor maneira de atribuirmos os identificadores aos periódicos e como validá-los junto ao site da CrossRef. Outros materiais e portais de periódicos foram pesquisados, dentre eles o Portal de Periódicos da UNICAMP (PPEC), que tem servido de modelo para a padronização de diversos elementos do PPUFGD. Recorremos ainda ao Boletim Técnico do PPEC, que apresenta um grande conjunto de pesquisas e manuais práticos aos editores e administradores de periódicos que utilizam o OJS.

\section{AÇÕES PARA A APLICAÇÃO E A VALIDAÇÃO DO DOI}

Inicialmente, houve a necessidade de configurar o plugin DOI no Portal a fim de gerenciar e usar os identificadores no próprio OJS. Após uma consulta feita aos diversos formatos de sufixos DOIs adotados por portais de outras instituições, optamos pela identificação onde consta o volume, seguido do ano e do ID do artigo conforme customização exibida na Figura 1 a seguir.

Figura 1 - Tela de configuração do plugin DOI no OJS.

\section{AGRARIAN}

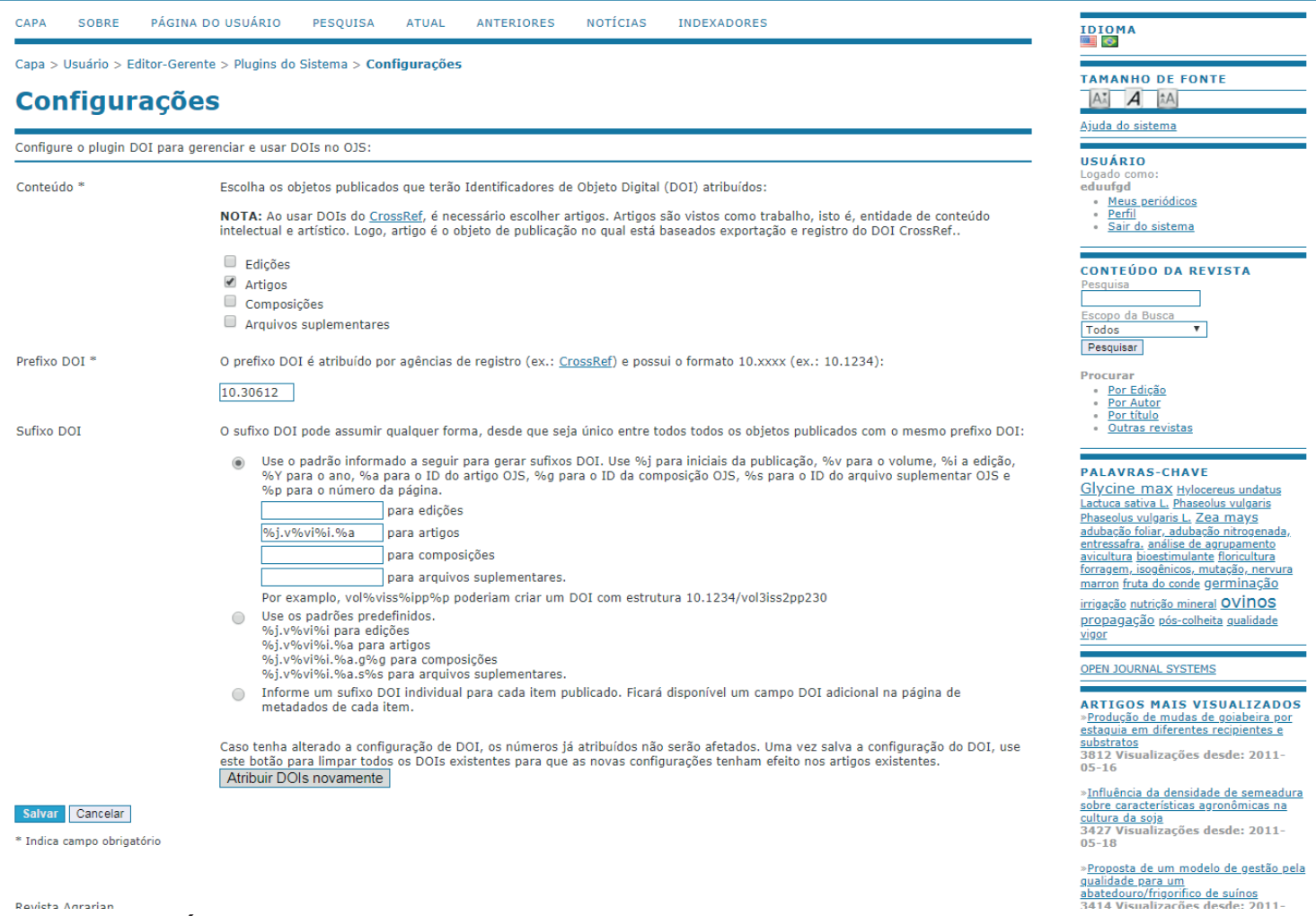

Fonte: Portal de Periódicos da UFGD, 2018.

A segunda etapa foi configurar o plugin para registro e exportação dos arquivos em XML (Extensible Markup Language) para a CrossRef. Não habilitamos o campo de registro automático, sendo assim, o procedimento que está em execução é o download 
dos XMLs de edições, que são obtidos pelo plugin "Registro e exportação XML CrossRef" na versão 2.4.8.3 do OJS.

Após exportar os artigos desejados para o formato CrossRef $X M L$, é feito o depósito (upload) desses arquivos no site da CrossRef. Os identificadores digitais para os artigos dos periódicos estão sendo depositados regularmente pela EdUFGD.

\section{NOVAS INDEXAÇÕES}

A Editora da UFGD vem almejando o aceite de novas indexações de seus periódicos em diretórios e base de dados nacionais e internacionais. Para tanto, iniciou um trabalho de conscientização dos editores através de treinamentos para que sejam padronizadas e atualizadas as informações sobre os periódicos, os seus respectivos metadados e o próprio layout dessas publicações (Figuras 2 e 3 ). O objetivo é adequar todos os periódicos às exigências dos diretórios e das bases de dados. A Editora também está realizando um trabalho de padronização dos metadados de números anteriores de todas as publicações hospedadas no Portal.

Figuras 2 e 3 - Treinamentos para editores de periódicos da UFGD.
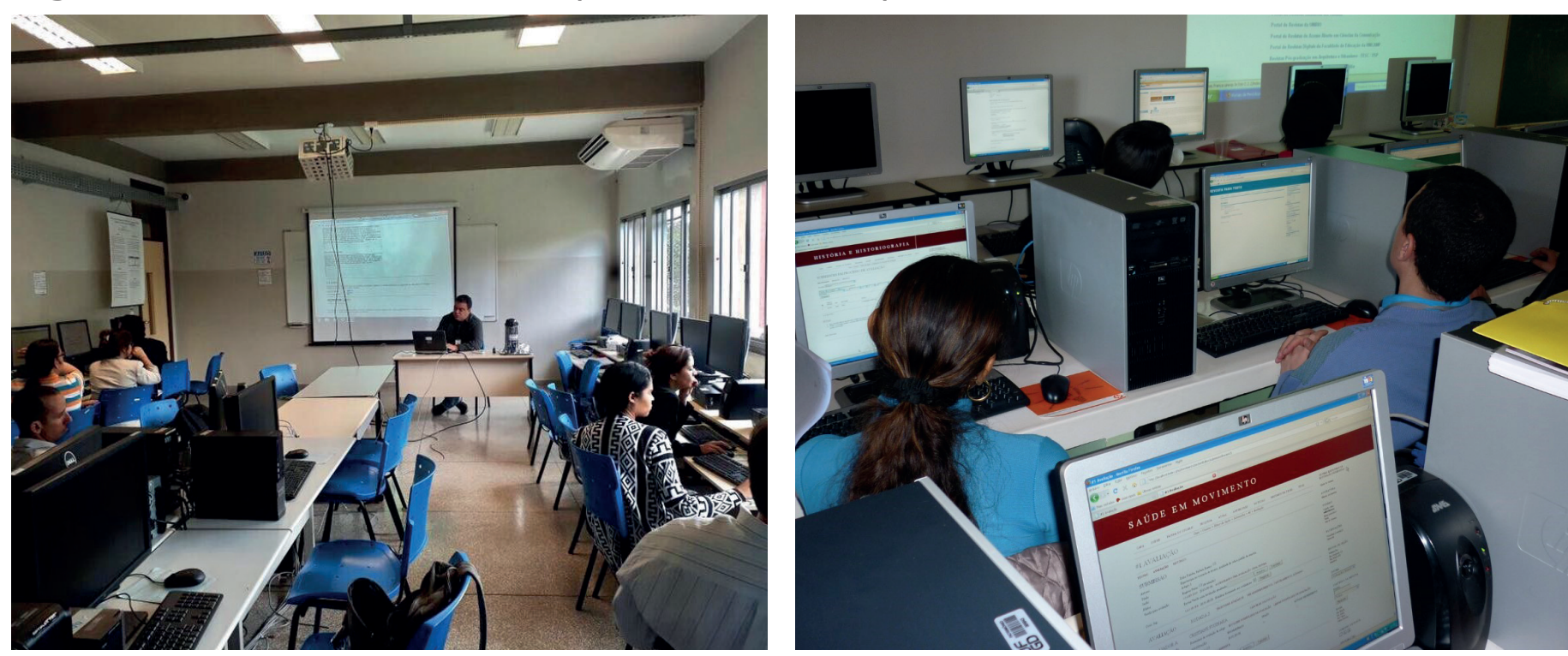

Fonte: Acervo da Editora da UFGD, 2018.

\section{RESULTADOS OBTIDOS E CONCLUSÃO}

Até junho de 2018, foram registrados e depositados 506 DOIs para artigos dos 16 periódicos hospedados no PPUFGD. Também foram solicitadas, pela própria Editora, indexações no Directory of Open Access Journals (DOAJ) e no Directory of Open Access Scholarly Resources (ROAD), as quais foram obtidas com sucesso para a maioria dos periódicos do PPUFGD, como podemos observar no Quadro 2:

Quadro 2 - Número de DOIs depositados e revistas indexadas no DOAJ e ROAD.

\begin{tabular}{|c|c|c|c|}
\hline Período & $\begin{array}{c}\text { DOIs depositados na } \\
\text { CrossRef }\end{array}$ & $\begin{array}{c}\text { Revistas indexadas no } \\
\text { DOAJ }\end{array}$ & $\begin{array}{c}\text { Revistas indexadas no } \\
\text { ROAD }\end{array}$ \\
\hline Março a junho/2018 & 506 & 12 & 16 \\
\hline
\end{tabular}

Fonte: Elaborado pelos autores, 2018. 
A Editora da UFGD está padronizando os metadados de todos os artigos publicados em seu Portal com o intuito de obtermos futuramente a indexação em outras bases de dados e diretórios importantes, tais como o REDIB, Dialnet e Redalyc.

Concluímos que a aplicação do DOI para as publicações no PPUFGD e a busca por novos indexadores será significativa para potencializar as publicações dos periódicos da instituição. Como o DOI passou a ser exigência de vários indexadores, os periódicos que são incluídos com sucesso nesses diretórios e bases de dados ganham mais visibilidade. Além disso, a atribuição de DOI e a aceitação de indexadores importantes também são fatores considerados nos sistemas de avaliação dos periódicos, como o Qualis, por exemplo, nos quais buscamos a melhoria na qualificação de nossas revistas.

\section{REFERÊNCIAS}

ASSOCIAÇÃO BRASILEIRA DE EDITORES CIENTÍFICOS. Especialistas destacam importância da obtenção do DOI via Crossref para potencializar publicações científicas. Sorocaba: Associação Brasileira de Editores Científicos, 2018. Disponível em: <https://www.abecbrasil.org.br/novo/2018/02/especialistas-destacamimportancia-da-obtencao-do-doi-via-crossref-para-potencializar-publicacoescientificas/>. Acesso em: 15 maio 2018.

BRITO, R. F. de, et al. Guia do usuário do Digital Object Identifier. Brasília: Instituto Brasileiro de Informação em Ciência e Tecnologia, 2015. Disponível em: <http://www.abecbrasil.org.br/arquivos/Guia usuario DOI-online3.pdf>. Acesso em: 20 fev. 2017.

BRITO, R. F. de; GUEDES, M. G.; SHINTAKU, M. Atribuição de identificadores digitais para publicações científicas: DOI para o SEER/OJS. Brasília: Instituto Brasileiro de Informação em Ciência e Tecnologia, 2013. Disponível em: <https:// econtents.bc.unicamp.br/boletins/index.php/ppec/article/view/6971>. Acesso em: 25 fev. 2018.

SANTOS, G. C. Como visualizar, coletar e incluir o DOI no Artigo: 9 passos básicos para o editor. Boletim Técnico do PPEC,Campinas-SP, v. 3, n. 1, 4 p., jan./abr. 2018. Disponível em: <https://econtents.bc.unicamp.br/boletins/index.php/ppec/ article/view/9155>. Acesso em: 15 mai. 2018.

SANTOS, G. C. Minicurso sobre preenchimento e edição de metadados no OJS para atribuição do DOI. Boletim Técnico do PPEC, Campinas-SP, v. 1, n. 1, 64 p., jan./ abr. 2016. Disponível em: <https://econtents.bc.unicamp.br/boletins/index. php/ppec/article/view/6979 >. Acesso em: 23 fev. 2018. 